\title{
O girino de Chiasmocleis alagoanus Cruz, Caramaschi \& Freire, 1999 (Anura: Microhylidae)
}

\author{
Filipe Augusto Cavalcanti do Nascimento ${ }^{1}$ \& Gabriel Omar Skuk ${ }^{2}$
}

Biota Neotropica v6 (n3) -http://www.biotaneotropica.org.br/v6n3/pt/abstract?short-communication+bn02506032006

\author{
Recebido em 10/05/2006. \\ Versão reformulada recebida em 30/09/2006 \\ Publicado em 26/10/2006
}
1Setor de Zoologia, Museu de História Natural, Universidade Federal de Alagoas, Av. Aristeu de Andrade, Farol, 57051- 090, Maceió,AL, Brasil.E-mail: filipe.bio@uol.com.br
${ }^{2}$ Departamento de Zoologia, Centro de Ciências Biológicas, Universidade Federal de Alagoas, praça Afrânio Jorge, s/nº, Prado, 57010-020, Maceió, AL, Brasil.E-mail: Gabrielskuk@terra.com.br

\begin{abstract}
Nascimento, F.A.C. and Skuk, G.O. The tadpole of Chiasmocleis alagoanus Cruz, Caramaschi \& Freire, 1999 (Anura: Microhylidae). Biota Neotrop. Sep/Dec 2006 vol. 6, no. 3 http://www.biotaneotropica.org.br/v6n3/pt/abstract?shortcommunication+bn02506032006 ISSN 1676-0603
\end{abstract}

Tadpoles of Chiasmocleis alagoanus were collected from a temporary pond in a remnant of Atlantic Forest, municipal district of Maceió, Alagoas, Brazil. The tadpole presents lentic and nektonic habit. Body triangular in lateral view and oval in dorsal view; snout truncate in lateral, dorsal and ventral view. Eyes lateral and nostrils absent. Spiracle medial with wide opening. Anal tube ventral and short, with central opening, without free margin. Oral opening terminal, with marginal papillae and jaw sheaths absent, with the superior semicircular labial flaps suspended on the extremities of the mouth. Tooth rows absent. Tail length representing $59.1 \%$ of total length. In preservative the body is brown light and the viscera is partially visible. Notes on the environment and comparison with other tadpoles of the genera are provided.

Key words: Anura, Microhylidae, larval morphology, Atlantic Rain Forest, Alagoas

\section{Resumo}

Nascimento, F.A.C. and Skuk, G.O. O girino de Chiasmocleis alagoanus Cruz, Caramaschi \& Freire, 1999 (Anura: Microhylidae). Biota Neotrop. Sep/Dec 2006 vol. 6, no. 3 http://www.biotaneotropica.org.br/v6n3/pt/abstract?shortcommunication+bn02506032006 ISSN 1676-0603

O girino de Chiasmocleis alagoanus é descrito pela primeira vez a partir de exemplares coletados na Mata do Catolé, município de Maceió, Alagoas, Brasil. O girino tem hábitos lêntico e nectônico. Corpo triangular em vista lateral e oval em vista dorsal; focinho truncado em vista lateral, dorsal e ventral. Olhos laterais e aberturas nasais ausentes. Espiráculo mediano e com ampla abertura. Tubo anal ventral curto com abertura central, sem margem livre. Abertura oral terminalmente localizada, sem peças queratinizadas. Abas labiais superiores semicirculares recobrindo parcialmente a abertura oral. Fileiras de dentículos córneos ausentes. Comprimento da cauda correspondendo a 59,1\% do comprimento total. Em preservativo o corpo é castanho claro e as vísceras parcialmente visíveis. Notas sobre o ambiente e comparação com outros girinos do gênero são apresentados.

Palavras-chave: Anura, Microhylidae, morfologia larval, Mata Atlântica, Alagoas

http://www.biotaneotropica.org.br 


\section{Introdução}

O gênero Chiasmocleis Méhely, 1904 é atualmente composto por 19 espécies, distribuídas desde o Panamá até o sudeste do Brasil, ocorrendo também ao norte e leste dos Andes (Canedo et al. 2004, Frost 2004). Nove dessas espécies são reconhecidas como habitantes da Floresta Atlântica brasileira (Cruz et al. 1997, 1999, Caramaschi \& Pimenta 2003, Canedo et al. 2004): C. alagoanus Cruz, Caramaschi \& Freire, 1999; C. atlantica Cruz, Caramaschi \& Izecksohn, 1997; C. capixaba Cruz, Caramaschi \& Izecksohn, 1997; C. carvalhoi Cruz, Caramaschi \& Izecksohn, 1997; C. cordeiroi Caramaschi \& Pimenta, 2003; C. crucis Caramaschi \& Pimenta, 2003; C. gnoma Canedo, Dixo e Pombal Jr., 2004; C. leucosticta (Boulenger, 1888) e C. schubarti Bokermann, 1952. Chiasmocleis alagoanus é a única espécie conhecida do gênero que habita remanescentes de Floresta Atlântica no estado de Alagoas, nordeste do Brasil. Cruz et al. (1999) incluíram essa espécie no grupo de C. schubarti, caracterizado pela ausência ou presença apenas vestigial de membranas interdigitais. Apenas três espécies de Chiasmocleis possuem girinos conhecidos: C. carvalhoi (Wogel et al. 2004) (também do grupo schubarti), C. cf. shudikarensis (Hero 1990) e C. ventrimaculata (Hero 1990, Schlüter \& Salas 1991). Apresentamos aqui a descrição do girino de $C$. alagoanus e comparamos com os girinos das espécies citadas acima.

\section{Material e Métodos}

Girinos foram colecionados na Mata do Catolé, remanescente de Floresta Atlântica situada no município de Maceió, Estado de Alagoas, Brasil (9³2’42,7" S e 3547’49,1" W), em março e junho de 2004. Os girinos coletados com redes de mão foram anestesiados em álcool a 10\% e conservados em formalina a $10 \%$ neutralizada com carbonato de cálcio, exceto dois exemplares que foram mantidos vivos em aquários até completarem a metamorfose para confirmação da identificação da espécie. A caracterização morfométrica foi baseada em duas séries de 6 girinos nos estágios 35-36 e 38-39 (Gosner 1960), respectivamente, com a terminologia e medidas seguindo Altig (1998) e Altig \& McDiarmid (1999), exceto o diâmetro interocular, em que foi considerado a distância entre as bordas internas dos olhos. Todas as medidas estão em milímetros e foram determinadas sob estereomicroscópio com retículo micrométrico exceto o comprimento total, largura e altura corporal, em que foi usado paquímetro de precisão 0,01 mm. Todo material foi depositado na coleção herpetológica do Museu de História Natural da Universidade Federal de Alagoas (MUFAL 3771, três exemplares; MUFAL 3772, dois exemplares e MUFAL 5277, 17 exemplares).

\section{Resultados e Discussão Descrição}

http://www.biotaneotropica.org.br
Girino no estágio 36 (MUFAL 5277; figuras 1 e 2). Corpo triangular em vista lateral e oval em vista dorsal e ventral; apresentando maior largura logo atrás dos olhos e maior altura no terço final do corpo; focinho truncado em vista lateral, dorsal e ventral. Olhos dispostos lateralmente, localizados no terço anterior do corpo, correspondendo a 40\% da distância olho-focinho e 18,9\% da distância interocular. Abertura das narinas ausentes. Espiráculo único, mediano, com uma abertura ampla, localizado no último terço do abdome e chegando a recobrir a abertura do tubo anal. Tubo anal curto, ventral, com abertura central dirigida ventroposteriormente, sem margem livre. Abertura oral terminalmente localizada, sem peças queratinizadas. Abas labiais semicirculares, pendentes sobre a abertura oral.

Comprimento da cauda correspondendo a 59,1\% do comprimento total. Musculatura caudal moderadamente desenvolvida, afilando gradativamente até a extremidade caudal, que apresenta flagelo. Altura máxima da membrana ventral pouco maior que a dorsal. Nadadeira dorsal com início na junção corpo-cauda, com inclinação de cerca de $30^{\circ}$, seguindo quase paralelamente ao eixo longitudinal da musculatura caudal ao longo do terço médio, passando a seguir levemente descendente até o início do terço final, convergindo abruptamente até o final da cauda. Membrana inferior com contorno retilíneo até a metade caudal, onde também passa a seguir convergentemente até a extremidade. Os dados de morfometria estão nas tabelas 1 e 2 .

Coloração em preservativo (formalina $10 \%$ neutralizada). Corpo castanho claro, com coloração mais acentuada em seu último terço; regiões dorsal e laterais com inúmeras pontuações, que aparecem em menor número na região ventral e são ausentes no terço final. Um par de manchas escuras em forma de arco é visível próximo a região interocular. Vísceras parcialmente visíveis. Membros e cauda seguindo o padrão corporal, borda da nadadeira ventral sem pigmentação.

\section{Ambiente e hábitos}

Os girinos de C. alagoanus foram coletados na localidade tipo da espécie (Cruz, Caramaschi e Freire 1999). Trata-se de um remanescente de Floresta Atlântica pertencente à região fitoecológica de Floresta Ombrófila, situado na faixa bioclimática de zero a noventa dias biologicamente secos (Assis 2000). Os girinos foram encontrados em uma poça temporária de tamanho aproximado de 25x10 m e profundidade máxima de 0,5 m no interior da mata; permaneciam a meia coluna d’água e nadavam ativamente; não formavam cardume. A água era relativamente límpida com muito sedimento no fundo. No mesmo local também foram encontrados girinos de Hylomantis sp. e Leptodactylus cf. labyrinticus. 


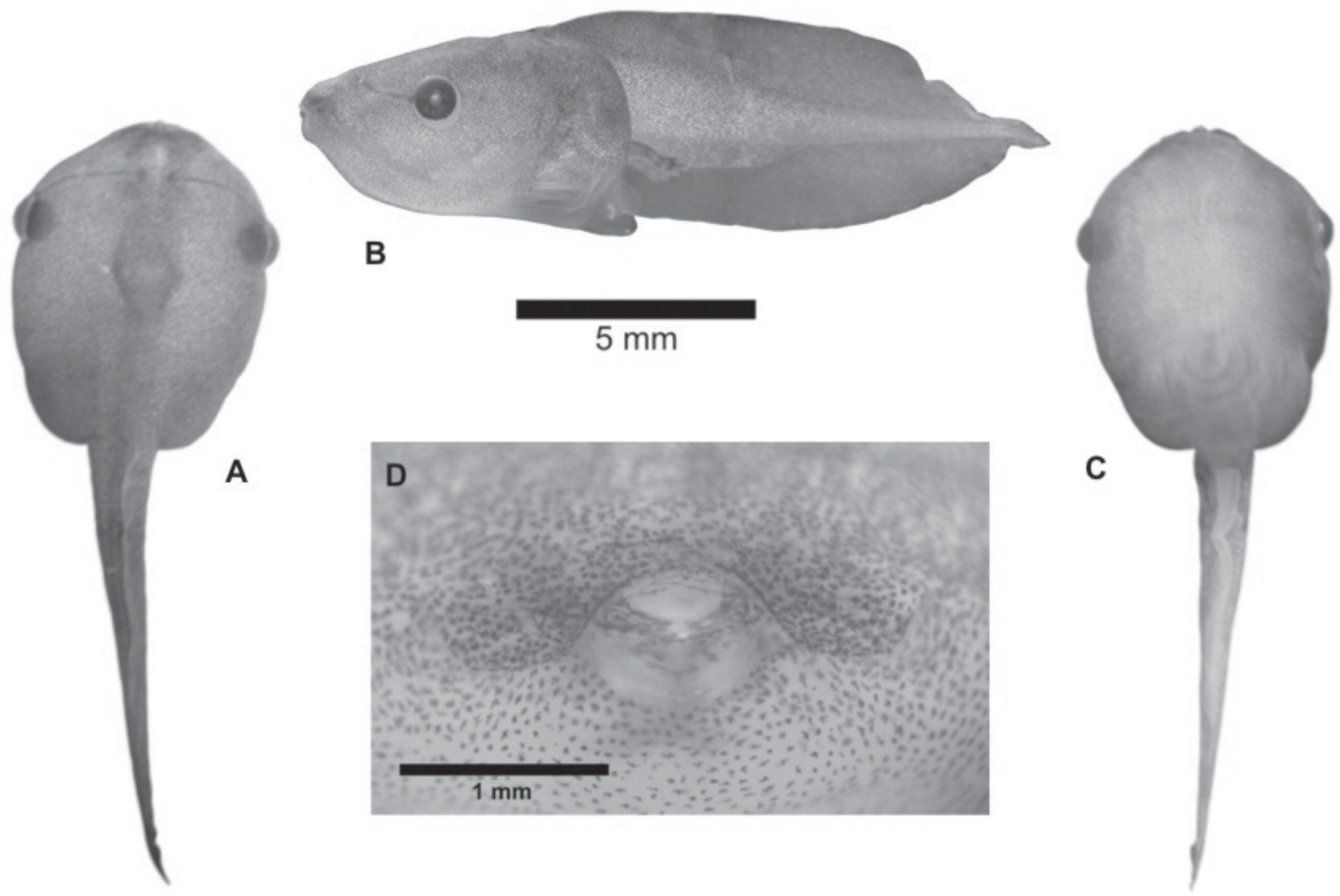

Figura 1. Girino de Chiasmocleis alagoanus (MUFAL 5277) no estágio 36 de Gosner (1960): (A) vista dorsal; (B) vista laterall; (C) vista ventrall; (D) disco oral.

Figure 1. Tadpole of Chiasmocleis alagoanus (MUFAL 5277) at stage 36 of Gosner (1960): (A) dorsal view; (B) lateral view; (C) ventral view; (D) oral disc.
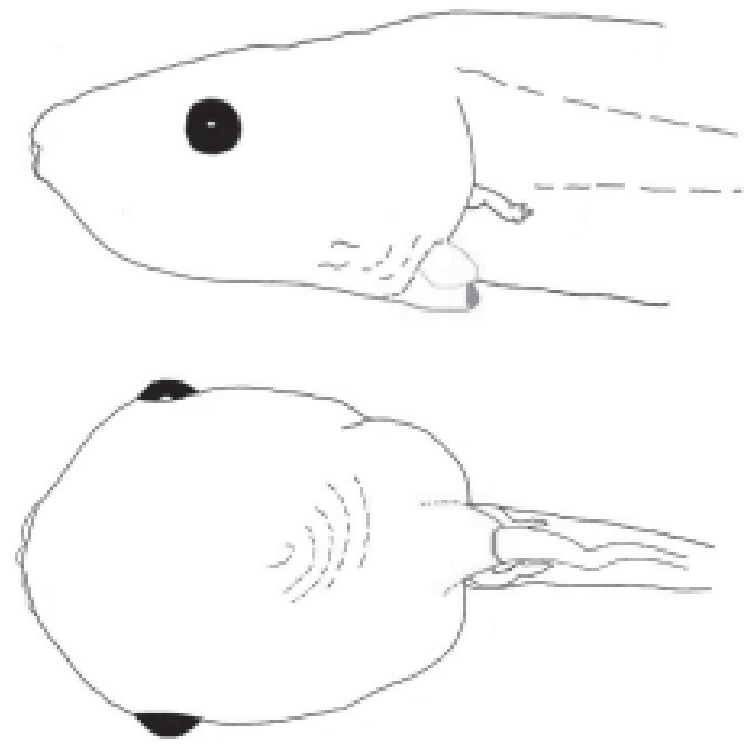

Figura 2. Girino de Chiasmocleis alagoanus (MUFAL 5277) no estágio 36 de Gosner (1960), evidenciando o espiráculo (em vermelho) e o tubo anal (em azul): (A) vista lateral; (B) vista ventral.

Figure 2. Tadpole of Chiasmocleis alagoanus (MUFAL 5277) at stage 36 of Gosner (1960) showing the spiracle (in red) and the anal tube (in blue): (A) lateral view; (B) ventral view.

http://www.biotaneotropica.org.br 
Tabela 1. Medidas (mm) de 6 girinos de Chiasmocleis alagoanus em estágio 35-36 (Gosner 1960).

Table 1 - Measurements ( $\mathrm{mm}$ ) of the 6 tadpoles of Chiasmocleis alagoanus at stage 35-36 (Gosner 1960).

\begin{tabular}{lcc}
\hline Medidas & Média \pm Desvio Padrão & Amplitude \\
\hline Comprimento total & $17,40 \pm 1,02$ & $16,00-18,50$ \\
Comprimento do corpo & $6,89 \pm 0,47$ & $6,20-7,50$ \\
Altura do corpo & $4,27 \pm 0,72$ & $3,10-5,10$ \\
Largura do corpo & $5,70 \pm 0,37$ & $5,10-6,20$ \\
Comprimento da cauda & $10,51 \pm 0,93$ & $9,46-11,90$ \\
Altura da cauda & $4,95 \pm 0,49$ & $4,10-5,50$ \\
Altura da musculatura caudal & $1,71 \pm 0,13$ & $1,50-1,90$ \\
Altura da membrana dorsal & $1,63 \pm 0,08$ & $1,60-1,80$ \\
Altura da membrana ventral & $2,00 \pm 1,11$ & $1,80-2,10$ \\
Diâmetro do olho & $0,95 \pm 0,05$ & $0,90-1,00$ \\
Distância interorbital & $5,26 \pm 0,29$ & $4,80-5,70$ \\
Distância olho-focinho & $2,46 \pm 0,29$ & $2,00-2,80$ \\
Largura do disco oral & $2,08 \pm 0,10$ & $1,90-2,20$ \\
\hline
\end{tabular}

Tabela 2. Medidas (mm) de 6 girinos de Chiasmocleis alagoanus em estágio 38-39 (Gosner 1960).

Table 2 - Measurements (mm) of the 6 tadpoles of Chiasmocleis alagoanus at stage 38-39 (Gosner 1960).

\begin{tabular}{lcc}
\hline Medidas & Média \pm Desvio Padrão & Amplitude \\
\hline Comprimento total & $19,24 \pm 1,19$ & $18,00-21,26$ \\
Comprimento do corpo & $6,86 \pm 0,63$ & $6,20-7,74$ \\
Altura do corpo & $3,84 \pm 0,59$ & $3,12-4,72$ \\
Largura do corpo & $5,11 \pm 0,49$ & $4,50-5,72$ \\
Comprimento da cauda & $12,40 \pm 1,14$ & $11,80-13,82$ \\
Altura da cauda & $4,22 \pm 0,62$ & $3,40-5,00$ \\
Altura da musculatura caudal & $1,60 \pm 0,24$ & $1,30-1,90$ \\
Altura da membrana dorsal & $1,47 \pm 0,26$ & $1,20-1,80$ \\
Altura da membrana ventral & $1,68 \pm 0,49$ & $1,00-2,40$ \\
Diâmetro do olho & $0,99 \pm 0,07$ & $0,90-1,10$ \\
Distância interorbital & $4,83 \pm 0,39$ & $4,60-5,60$ \\
Distância olho-focinho & $2,24 \pm 0,31$ & $1,90-2,80$ \\
Largura do disco oral & $2,08 \pm 0,10$ & $1,90-2,20$ \\
\hline
\end{tabular}

http://www.biotaneotropica.org.br 


\section{Comparação com outras espécies}

A morfologia larval de $C$. alagoanus mostrou-se muito similar a dos girinos conhecidos deste gênero. Não houve um caráter diagnóstico seguro que distinguisse os girinos de $C$. alagoanus dos de $C$. carvalhoi descritos por Wogel et al. (2004), apesar disso os girinos de C. alagoanus apresentaram comprimento total médio um pouco maior que os de C. carvalhoi (17,4 contra 16,6 mm, estágio 35-36). No girino de $C$. ventrimaculata a nadadeira dorsal se origina após o final do corpo e a parte distal da cauda é curvada dorsalmente (Schlüter \& Salas 1991), enquanto que em $C$. alagoanus a nadadeira dorsal teve início no final do corpo e a parte distal da cauda apresentou-se retilínea. O girino de C. cf. shudikarensis não foi descrito com detalhes suficientes para permitir uma comparação segura (Hero 1990).

Apesar da heterogeneidade morfológica que envolve os adultos do gênero Chiasmocleis (Caramaschi e Cruz 2001), os girinos parecem ter uma grande similitude quanto à forma. Porém, faz-se necessário conhecer os girinos das demais espécies, a fim de que mais informações estejam disponíveis para o esclarecimento das relações taxonômicas dentro do gênero.

\section{Agradecimentos}

À Fundação de Amparo à Pesquisa do Estado de Alagoas (FAPEAL) pelo auxílio financeiro (processos $n^{\circ}$ 2002.08.110-02 e 2004.04.29864-02). A Ubiratan Gonçalves pela ajuda nas coletas de campo, e a Selma Torquato pelo auxílio nos trabalhos de laboratório.

\section{Referências Bibliográficas}

ALTIG, R. 1998. A key of the tadpoles of the continental United States and Canada. Contemporary Herpetology Information Series. URL: $\underline{\text { http:// }}$ dataserver.calacademy.org/herpetology/herpdocs/chis/ 1998/2/. Acesso em outubro 2003.

ALTIG, R. \& MCDIARMID, R. W. 1999. Body plan: development and morphology. Pp. 24-51. In. Tadpoles: The Biology of Anuran Larvae (R.W. McDiarmid \& R. Altig, eds.). Chicago and London. The University of Chicago Press.

ASSIS, J. S. 2000. Biogeografia e conservação da biodiversidade - projeções para Alagoas. Maceió-São Paulo. Edições Catavento. 200pp.

CANEDO, C., DIXO, M. \& POMBAL JR., J. J. 2004. A new species of Chiasmocleis Méhelÿ, 1904 (Anura, Microhylidae) from the Atlantic RainForest of Bahia, Brazil. Herpetologica, 60(4): 495-501.

CARAMASCHI, U. \& CRUZ, C. A. G. 2001. A new species of Chiasmocleis Méhelÿ, 1904 from brazilian amazonia (Amphibia, Anura, Microhylidae). Bol. Mus. Nac., N.S., Zool. Rio de Janeiro, 469:1-8.
CARAMASCHI, U. \& PIMENTA, B. V. S. 2003. Duas novas espécies de Chiasmocleis Méhely, 1904 da Mata Atlântica do Sul da Bahia, Brasil (Amphibia, Anura, Microylidae). Arq. Mus. Nac., 61: 195-202.

CRUZ, C. A. G., CARAMASCHI, U. \& FREIRE, E. M. X. 1999. Ocurrence of the genus Chiasmocleis (Anura: Mycroylidae) in the State of Alagoas, north-eastern Brazil, with a description of a new species. J. Zool. Lond., 249: 123-126.

CRUZ, C.A. G., CARAMASCHI, U. \& IZECKSOHN, E. 1997. The genus Chiasmocleis Méhelÿ, 1904 (Anura, Microhylidae) in the Atlantic Rain Forest of Brazil, with description of three new species. Alytes, 15(2): 49-71.

FROST, D. R. (ed.). 2004. Amphibiam Species of the Worldan on line reference. V 3.0. URL: http://research.amnh.org/ herpetology/amphibia/index.php. Acesso em julho 2005.

GOSNER, K. L. 1960. A simplified table for staging anuran embryos and larvae with notes on identification. Herpetologica, 16:183-190.

HERO, J. M. 1990. An illustrated key to tadpoles occurring in the Central Amazon rainforest, Manaus, Amazonas, Brasil. Amazoniana, 11:201-262.

SCHLÜTER, A. \& SALAS, A. W. 1991. Reproduction, tadpoles, and ecological aspects of three syntopic microhylid species from Peru (Amphibia: Mycrohylidae). Stuttgarter Beitr. Naturk., Serie A, 458:1-17.

WOGEL, H., ABRUNHOSA, P. A. \& PRADO, G. M. 2004. The tadpole of Chiasmocleis carvalhoi and the advertisement calls of three species of Chiasmocleis (Anura, Microhylidae) from the Atlantic rainforest of southeastern Brazil. Phyllomedusa, 3(2): 133-140.

Título: O girino de Chiasmocleis alagoanus Cruz, Caramaschi \& Freire, 1999 (Anura: Microhylidae).

Autores: Nascimento, F.A.C. and Skuk, G.O.

Biota Neotropica, Vol. 6 ( número 3 ): 2006

http://www.biotaneotropica.org.br/v6n3/pt/ abstract?short-communication+bn02506032006

Recebido em 10/05/2006.

Versão reformulada recebida em 30/09/2006

Publicado em 26/10/2006

ISSN 1676-0603 